\title{
Un facsimile non è un template... ma sono entrambi invariabili?
}

Sara Giovine

PUBBLICATO: 29 GIUGNO 2021

\section{Quesito:}

Rispondiamo ai numerosi lettori che ci hanno scritto per conoscere la differenza di significato tra facsimile e template riproponendo la risposta di Gabriella Cartago, pubblicata sul n. 58 (I, 20I9) della nostra rivista La Crusca per voi. Aggiungiamo inoltre una breve nota per rispondere a quanti ci chiedono quale sia la corretta forma di plurale di facsimile.

\section{Un facsimile non è un template... ma sono entrambi invariabili?}

I madrelingua inglesi probabilmente risponderebbero: "No, facsimile e template non sono sinonimi". I loro dizionari, in effetti, dentro ampi ventagli di sinonimi dell'uno non riportano mai l'altro. Si veda l'OED per facsimile: "copy, reproduction, duplicate, photocopy, mimeograph, mimeo, replica, likeness, carbon, carbon copy, print, reprint, offprint, image; double, lookalike, twin, clone, duplicate, perfect likeness, exact likeness, echo, replica, copy, imitation, picture, image, living image, mirrorimage". E per template: "cast, die, form, matrix, shape, container".

In italiano, però, in cui entrambe le voci arrivano dall'inglese, qualche sovrapposizione di significato può crearsi. È facsimile, ambientatosi nella nostra lingua molto prima (addirittura nell'Ottocento) a invadere la sfera d'uso che spetterebbe più propriamente a template, di approdo recente, nella seconda metà del Novecento.

Facsimile significa, a norma di vocabolari, 'copia, riproduzione esatta di un originale' (Zingarelli 20r9, Sabatini-Coletti 2008, Hoepli 2018, Devoto-Oli 2018, Garzanti 2017). Se scorriamo i giornali degli ultimi due o tre anni, troviamo i facsimile più disparati: Di Battista che straccia in tv un facsimile di 5.0oo franchi africani; un fac-simile di vestiario dei deportati nei lager ispirato a fotografie d'epoca; un facsimile del celebre doppio ritratto dei duchi di Urbino; biglietti da Io euro "fac-simile" in grado di ingannare i cambiavalute automatici. Come si vede nell'ultimo caso, la piena e secolare acclimatazione di facsimile negli usi italiani è tale da renderne possibile anche l'estensione metaforica (virgolettata). L'estensione metaforica non manca, peraltro, di essere puntualmente registrata nei vocabolari ('esempio di imitazione o somiglianza', 'persona o cosa tanto simile a un'altra da sembrarne la copia' e cosi via). Ma facsimile si usa anche con un valore sovrapponibile a quello di template, nuovo arrivato, in età digitale, a occupare uno spazio rinnovato. Sempre nella stampa quotidiana dei nostri giorni troviamo, infatti: "Il bando ed il fac-simile di domanda sono pubblicati nel sito web del Centro Studi"; "un facsimile del modello di autocertificazione da presentare alle scuole per le famiglie lombarde alle prese con l'obbligo dei vaccini per l'iscrizione a scuola". Nel significato, dunque, di 'modello'.

Modello è, di fatto, il valore che viene attribuito a template nei dizionari italiani dell'uso generale che lo registrano, come i seguenti:

- Zingarelli 20I9: template "documento con parti già compilate e altre da compilare a cura dell'utente / nella programmazione di pagine web, modello grafico predefinito utilizzabile per la 
messa in rete di contenuti" (1984).

- Devoto-Oli 20I8: template "inform. Modello predefinito che consente di creare o inserire contenuti di diverso tipo in un documento o una pagina web (1996) propriam. sagoma, modello".

Dunque, modello, ma, come si vede, in senso puramente formale, indipendente dal contenuto, privo di contenuto riproducibile, sinonimo, semmai, diformat (un altro anglicismo), ossia formato, disposizione prefissata. Proprio perché ancora non altrettanto familiare a lettori e parlanti italiani, nella stampa si è incontrato spesso accompagnato da spiegazioni, che ruotano intorno a modello standard, schema, procedura. Per esempio: «abbiamo introdotto dei cosiddetti "template" ossia dei modelli standard, delle procedure in gran parte automatizzate, per tipologia di settore e di imprese».

Nondimeno template ha, a sua volta, iniziato a conoscere impieghi metaforici: «Quello che è avvenuto nei zo minuti del volo fra il Vaticano e l'eliporto di Albano, accanto a Castel Gandolfo, nell'elicottero troppo lontano per battere quel ritmo angoscioso che la storia contemporanea e il cinema ci hanno martellato in testa come segni apocalittici, sarà il "template", il calco, la sagoma sui quali ogni futuro Sommo Pontefice dovrà plasmare e chiudere il proprio regno». Dove la sovrapponibilità con facsimile è piuttosto evidente.

Se, dunque, schematizzando, nel facsimile di un modello di autocertificazione si indicheranno i tipi di dati da inserire, il template del medesimo non differirà di molto (sempre, naturalmente, che non si tratti di usi figurati). Non dovrà specificare alcun elemento di contenuto, ma si presenterà con indicazioni relative alla loro sistemazione nel documento e, soprattutto, ne sarà lo scheletro virtuale, una sorta di realizzazione precostituita. Può aiutare a distinguerlo da template (modello "dinamico" dentro cui gli utenti inseriscono i propri dati), ricordare che facsimile ha generato, per riduzione, fax, servizio che trasmette copie di immagini "fisse". In definitiva, facsimile va inteso come "copia di..., template come 'modello su cui...'.

Gabriella Cartago

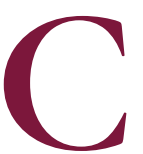

hiarito l'esatto valore semantico del sostantivo facsimile, veniamo ora alla formazione del suo plurale, che ha suscitato più di un dubbio nei nostri lettori, incerti sulla declinabilità del sostantivo; prima di rispondere è però necessaria qualche osservazione preliminare sulla struttura morfologica della voce in questione.

La forma facsimile, attestata anche nelle varianti con grafia analitica fac-simile e fac simile (oltre che in quella con assimilazione consonantica fassimile, che risulta però ormai rara e desueta), è una parola composta formata dal verbo latino fac 'fai' (imperativo di facěre 'fare') e dall'aggettivo sostantivato simüle 'il simile'. Come rilevato già da Migliorini 1948 , p. 77, si tratta di una formazione interpretabile come uno pseudolatinismo, ossia una parola formata in epoca moderna a partire da elementi latini (come referendum, factotum e vademecum), attestata in italiano dal I822 (nella variante fac-simile, cfr. DELI): con una ricostruzione ripresa dai principali dizionari etimologici, Benedetti I974, p. II ha ipotizzato in particolare che la voce sia stata coniata in Inghilterra alla fine del Seicento e che da li si sia poi diffusa in altre lingue, "agevolata dalla struttura latina nel suo processo di circolazione europea".

Al plurale, il sostantivo oscilla da sempre tra la forma invariabile (i facsimile) e quella in -i (i facsimili), e la ragione è da ricercare proprio nella particolare natura del composto: in qualità di pseudolatinismo, la forma dovrebbe infatti restare invariata al plurale, secondo il trattamento 
riservato di norma ai latinismi di origine moderna (per esempio il referendum > i referendum); se invece la consideriamo appartenente alla categoria dei composti Verbo + Sostantivo (considerazione facilitata dalla coincidenza tra il simüle latino e il corrispondente aggettivo italiano), seguendo le norme che regolano la formazione del plurale di tali composti, ne dovremmo declinare il secondo elemento componente, modificando quindi la sola desinenza finale (si veda anche la scheda Plurale di alcuni nomi composti).

Come indicato nelle principali grammatiche dell'uso (tra cui Serianni I989, III, pp. I45-47), per i composti formati da una base verbale e da un sostantivo, si profilano infatti tre possibilità nel passaggio dal singolare al plurale:

- se il sostantivo è plurale, il composto resta invariato (per esempio il battipanni > i battipanni, il guastafeste > i guastafeste, il portapenne > i portapenne);

- se il sostantivo è singolare e di genere maschile, viene declinato al plurale solo quest'ultimo (per esempio il parafango > i parafanghi, il passaporto > i passaporti, il passatempo > $i$ passatempi; seguono inoltre la stessa regola anche i composti con una base verbale unita al sostantivo femminile mano, a causa della desinenza in -o, per esempio l'asciugamano $>$ gli asciugamani, il corrimano $>i$ corrimani);

- se infine il sostantivo è singolare e di genere femminile, il composto resta invariato al plurale (per esempio il cavalcavia > $i$ cavalcavia, il posacenere $>i$ posacenere, il portabandiera $>i$ portabandiera).

Sulla base di tale norma, il composto facsimile, che contiene al suo interno un sostantivo di genere maschile, dovrebbe formare il proprio plurale modificando la desinenza finale (e dunque $i$ facsimili), ma, come si è già anticipato, nell'italiano corrente l'uso di tale forma convive con quella invariabile: si tratta di unoscillazione attestata nella nostra lingua già dalla metà dell'Ottocento, come ci documentano alcune delle occorrenze registrate dal principale dizionario storico dell'italiano, il GDLI. Per esempio:

Ritengo ancora le carte famose, per due ragioni: l'una perché voglio farne cavare alcuni fac-simile, e l'altra perché temo che, durante la stampa, occorra di verificare qualche cosa sugli originali. (C. Cattaneo, Epistolario, I850)

Soprattutto aveva un'attitudine specialissima ad imitare altrui; e copiava le scritture d'ogni genere da parer fac-simili i più perfetti. (G. Rovani, Cento anni, 1869 )

Nell'uso contemporaneo, pur nella coesistenza delle due forme, appare tuttavia largamente prevalente il ricorso al plurale invariato: una ricerca nelle pagine italiane di Google (condotta l'iı/I2/20) restituisce infatti 37.900 occorrenze della stringa di ricerca "i fac-simile" (con o senza trattino) e I9.600 di "i facsimile" (con grafia univerbata) contro i 17.800 di "i fac-simili" e i 7.520 di "i facsimili".

Di fronte a una simile oscillazione, i lessicografi non hanno assunto una posizione unanime: il Garzanti 2017 e il DOP ammettono infatti il solo plurale in -i (nel caso del DOP segnalando esplicitamente come errata la forma invariabile); anche il GRADIT registra facsimile semplicemente come sostantivo maschile (e infatti nell'edizione in chiave USB del 2007 si legge il plurale facsimili), al contrario del Sabatini-Coletti e del Devoto-Oli 202I, che lo registrano come invariabile; infine lo Zingarelli 202I ritiene accettabili entrambe le forme di plurale.

Concludendo, per le ragioni storico-morfologiche che si sono viste, si possono considerare corrette entrambe le forme di plurale. 
Nota bibliografica:

- Anna Benedetti, Le traduzioni italiane da Walter Scott e i loro anglicismi, Firenze, Olschki, I974.

- Bruno Migliorini, Latinismi recenti nel lessico europeo, in Id., Lingua e cultura, Roma, Tumminelli, I948, pp. 75-8I.

\section{Cita come:}

Sara Giovine, Un facsimile non è un template... ma sono entrambi invariabili? , "Italiano digitale", XVII, 2021/2 (aprile-giugno)

DOI: $10.35948 / 2532-9006 / 2021.9562$

Copyright 2021 Accademia della Crusca

Pubblicato con licenza creative commons CC BY-NC-ND 